\title{
Distribution of sialic acid receptors and experimental infections with different subtypes of influenza A viruses in Qinghai-Tibet plateau wild pika
}

Yan $\mathrm{Li}^{1+}$, Haixia Xiao ${ }^{2+}$, Chaobin Huang ${ }^{3}$, Haigang Sun ${ }^{4}$, Laixing $\mathrm{Li}^{5}$, Jingliang Su${ }^{4}$, Juncai Ma ${ }^{6}$, Di Liư ${ }^{6}$, Han Wang ${ }^{1}$, Wenjun Liu', George F Gao ${ }^{1,2,4,7,8}$, Xiangdong $\mathrm{Li}^{3^{*}}$ and Jinghua Yan ${ }^{1 *}$

\begin{abstract}
Background: The plateau pika (Ochotona curzoniae) is a small rabbit-like mammal that lives at high altitudes in the Qinghai-Tibet plateau and is in close contact with birds. Following the outbreak of highly pathogenic avian influenza (HPAl) H5N1 during 2005 in the migratory birds of Qinghai Lake, two clades of H5N1 have been found in pikas. However, the influenza virus receptor distribution in different tissues of this animal and its susceptibility to influenza A viruses have remained unclear.

Methods: The sialic acid receptor distribution tropism in pika was investigated using fluorescent Sambucus nigra and biotinylated Maackia amurensis I and II. Furthermore, the replication of three influenza A viruses H1N1, H3N2, and H5N1 in this animal was examined by immunohistochemistry and RT-PCR. Morphological and histopathological changes caused by infection were also analyzed with hematoxylin and eosin ( $\mathrm{H}$ \& E) staining.

Results: Human influenza virus-recognizing SAa2,6Gal receptors are widely expressed in the lung, kidney, liver, spleen, duodenum, ileum, rectum, and heart, whereas avian influenza virus-recognizing SAa2,3Gal receptors are strongly expressed in the trachea and lung of pika. M1 could be detected in the lungs of pikas infected with H1N1, H3N2, and H5N1 by either immunostaining or RT-PCR, and in the brain of H5N1-infected pikas. Additionally, three subtypes of influenza A viruses were able to infect pika and caused varying degrees of pneumonia with epithelial desquamation and alveolar inflammatory cell infiltration. Slight pathological changes were observed in H1N1-infected lungs. A few small bronchi and terminal bronchioles were infiltrated by lymphocytic cells in H3N2-infected lungs. In contrast, serious lung damage, such as alveolar capillary hyperemia, edema, alveolar collapse, and lymphocytic infiltrations was observed in $\mathrm{H} 5 \mathrm{~N} 1$-infected group. Furthermore, neural system changes were present in the brains of H5N1-infected pikas.
\end{abstract}

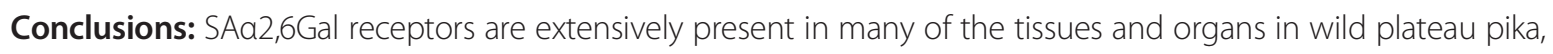
whereas SA2,3Gal-linked receptors are dominant on the tracheal epithelial cells. H1N1, H3N2, and H5N1 were able to infect pika and caused different degrees of pathogenic changes in the lungs. Altogether, these results suggest that wild pika has the potential to be a host for different subtypes of influenza A viruses.

Keywords: Sialic acid receptors, Experimental infection, Influenza A viruses, Wild plateau pika, Pathogenic changes

\footnotetext{
* Correspondence: xiangdongli68@126.com; yanjh@im.ac.cn

${ }^{\dagger}$ Equal contributors

${ }^{3}$ State Key Laboratory of the Agro-Biotechnology, College of Biological Sciences, China Agricultural University, Beijing 100193, China

${ }^{1}$ CAS Key Laboratory of Pathogenic Microbiology and Immunology, Institute of Microbiology, Chinese Academy of Sciences, Beijing 100101, China

Full list of author information is available at the end of the article
}

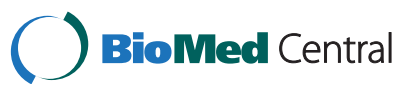

(c) 2015 Li et al.; licensee BioMed Central. This is an Open Access article distributed under the terms of the Creative Commons Attribution License (http://creativecommons.org/licenses/by/4.0), which permits unrestricted use, distribution, and reproduction in any medium, provided the original work is properly credited. The Creative Commons Public Domain Dedication waiver (http://creativecommons.org/publicdomain/zero/1.0/) applies to the data made available in this article, unless otherwise stated. 


\section{Background}

Influenza A viruses are important pathogens in the poultry industry and also pose a large threat to public health. Sixteen hemagglutinin (HA) subtypes and nine neuraminidase (NA) subtypes of influenza A viruses are found in waterfowl [1], which are considered the natural reservoir of influenza A viruses. Moreover, two novel bat-derived subtypes of influenza A viruses, H17N10 and H18N11, were recently discovered respectively in little yellow-shouldered bats (Sturnira lilium, family Phyllostomidae) and flat-faced fruit bats (Artibeus planirostris) by sequencing analyses [2-4]. However, as yet, those two novel influenza viruses lack classical functions for the viral envelope proteins, the HA cannot bind to any traditional influenza virus receptors, and NA loses the neuraminidase activity to remove sialic acid [3-8]. The discovery of influenza-like viruses in bats has highlighted the concerns of the scientific community over a possible broader host range of the virus.

As stated above, migratory and wild birds (in particular, waterfowl) are generally considered to be the natural hosts for all known subtypes of influenza A viruses, with some subtypes being more adapted to infect humans, birds, pigs, horses, and other animals. Certain subtypes of influenza A viruses that have adapted to infect humans are currently circulating among the human population worldwide, e.g., the $\mathrm{H} 1 \mathrm{~N} 1$ and $\mathrm{H} 3 \mathrm{~N} 2$ viruses. Whereas some other HA subtypes of avian influenza virus, such as $\mathrm{H} 5, \mathrm{H} 6, \mathrm{H} 7, \mathrm{H} 9$, and $\mathrm{H} 10$, have crossed the interspecies barrier and have acquired the ability to infect humans but lack the ability to circulate among humans. Human infections with such avian influenza viruses have ranged from mild symptoms (e.g., conjunctivitis and influenza-like illness) to severe disease, including deaths [9-18]. Worryingly, HPAI virus $\mathrm{H} 5 \mathrm{~N} 1$ and the low pathogenic avian influenza (LPAI) A virus H7N9 have caused a great number of human infections and deaths. According to the records from the World Health Organization (WHO), H5N1 influenza has caused 650 human infections, with 386 confirmed deaths, from to 2003 to January 24, 2014 [19]. The novel H7N9 virus that emerged in March 2013 has caused 419 infections and 127 deaths until July, 2014 [20].

Waterfowl and shorebirds have historically been considered the primary natural reservoir for influenza viruses, allowing their circulation with low pathogenicity among the flock. However, the outbreak of H5N1HPAI at Qinghai Lake astonished the world with the death of over 6,000 migratory birds in 2005 [21,22]. Since 2005, H5N1 has spread, following the migration routes of migratory birds, to Europe and some parts of Africa, and has caused severe poultry outbreaks and human infections [23]. In addition, H5N1 has been identified in the plateau pikas [24], which are found at the Qinghai-Tibet plateau and share their local environment with a plethora of wild birds. Consequently, concerns have been raised as to whether pika is a novel natural host of H5N1 and/or other subtypes of influenza viruses.

A major determinant of infection is the presence of sialosaccharide receptors on the host cell surface to which viral HA can bind. Avian influenza viruses preferentially bind to sialic acid (SA) receptors that are linked to galactose by an $\alpha 2,3$ linkage (SA $2,3 \mathrm{Gal})$, while human and classical swine viruses show preference for receptors with an $\alpha 2,6$ linkage $(\mathrm{SA} \alpha 2,6 \mathrm{Gal})[25,26]$. Distribution tropisms of receptors among different hosts could account for variation in their susceptibility to infection by influenza viruses. The distribution of the influenza virus receptors $\mathrm{SA} \alpha 2,3 \mathrm{Gal}$ and $\mathrm{SA} \alpha 2,6 \mathrm{Gal}$ has been assessed in several avian and mammal species, and the distribution patterns vary among different species [26-35]. As an important member of the Qinghai-Tibet plateau ecosystem, plateau pika was found to be the host of H5N1 influenza viruses [24]. However, little is known about influenza virus receptor distribution in this animal and the pathogenicity caused by H5N1 infection. Thus, we explored the influenza virus receptor distribution in different organs of pika and investigated the susceptibility of the plateau pika to a variety of subtypes of influenza A viruses (including H1N1, H3N2, and H5N1) in this study.

\section{Results}

Distribution of SAa2,3Gal and SAa2,6Gal receptors in the different organs of pika

To evaluate the potential role of receptor distribution in the susceptibility of pika to influenza virus infection, we investigated the distributions of SA $\alpha 2,3 \mathrm{Gal}$ and SA $\alpha 2,6 \mathrm{Gal}$ receptors in various organs, such as the trachea, lung, duodenum, ileum, rectum, heart, liver, kidney, brain, and spleen, by using biotinylated Maackia amurensis (MAA) lectin (red) I (MAAI) and II (MAAII) for SA 2,3Gal binding and FITC-labeled Sambucus nigra (SNA) lectin

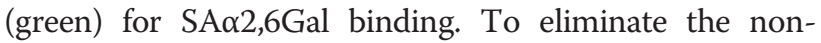
specific staining of MAAI, MAAII and SNA lectins, we used human trachea exclusively expressing SA 2 2,6Gal receptor and duck duodenum with only SA 2 2,3Gal expression to determine the background levels of lectin staining. It was clear that these three lectins displayed very low background staining (Additional file 1: Figure S1).

Based on this result, we performed lectin staining in the different tissues and organs of pika. Individual staining with DAPI, SNA, MAAI, and MAAII for different tissues was shown in (Additional file 2: Figure S2). And those overlapping images for different staining combinations were clearly presented in Figure 1. We observed strong red (MAAI and MAAII) and weak green staining at the tip of the ciliated columnar epithelium, suggesting that $\mathrm{SA} \alpha 2,3 \mathrm{Gal}$ receptors rather than $\mathrm{SA} \alpha 2,6 \mathrm{Gal}$ are 


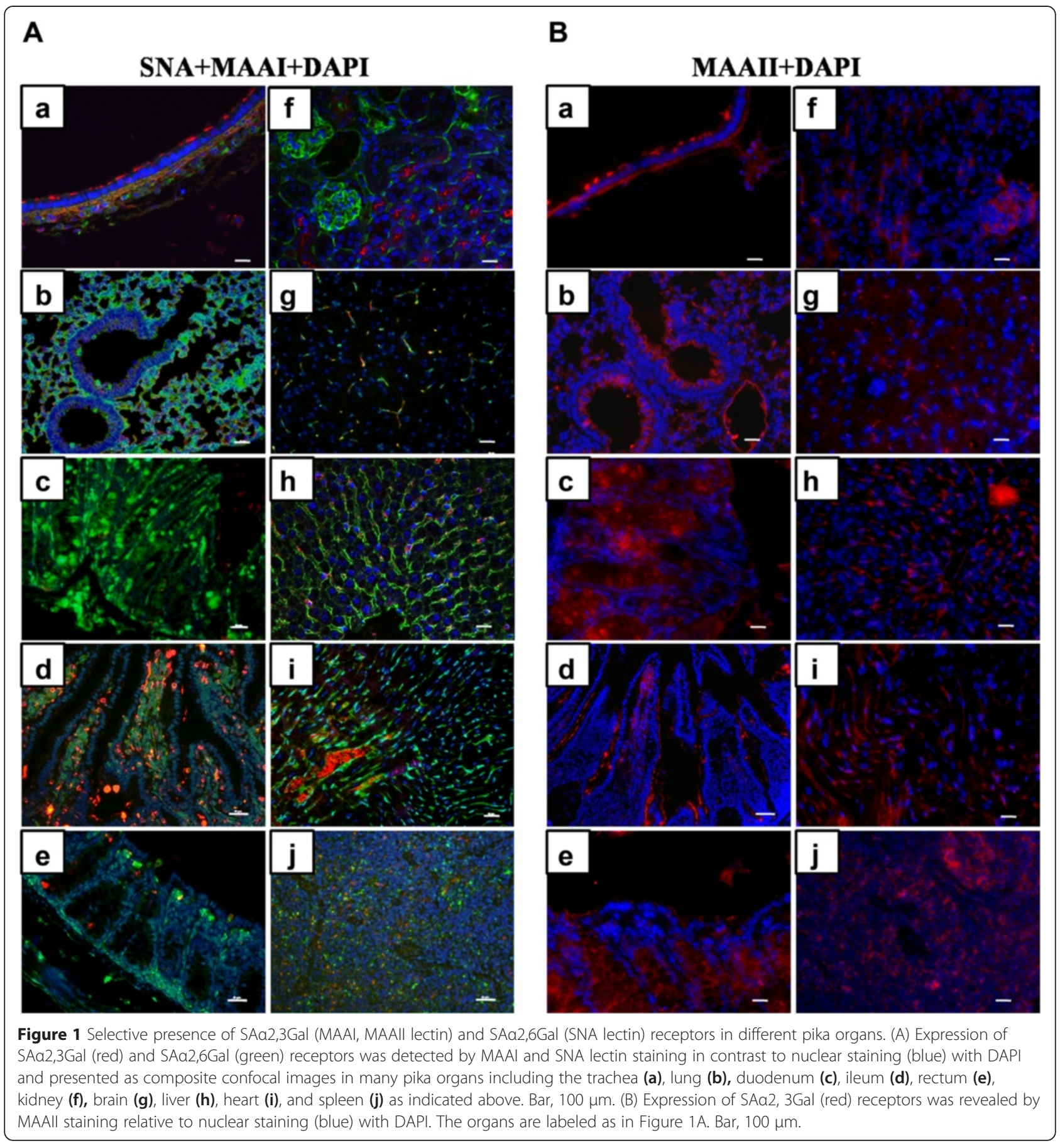

widespread on the tracheal epithelial cells (Figure 1A-a and $\mathrm{B}-\mathrm{a}$ ). We also found that the tracheal lamina propria and mucous glands express both SA $\alpha 2,3 \mathrm{Gal}$ and SA $\alpha 2,6-$ Gal linked receptors when MAAI, MAAII, and SNA were used for staining (Figure 1A-a and B-a). However, alveolar cells are rich in SA $2,6 \mathrm{Gal}$ receptors and also have a strong staining with MAAII lectin but have a faint MAAI signal (Figure 1A-a and B-a). In the lower respiratory tract, alveoli cells display predominant expression of SA 2 , $6 \mathrm{Gal}$ receptors. Contrary to the findings in chickens (Gallus gallusdomesticus) and ducks (Anas) [28], very few epithelial cells were positive for either MAAI or SNA staining in the smaller and terminal bronchiole of pika (Figure 1A-b). In contrast to MAAI, MAAII lectin was abundant in the smaller and terminal bronchiole of this animal (Figure 1B-b). The fluorescent staining along the epithelial lining of the mucosa demonstrated the presence of SA $\alpha 2,6 \mathrm{Gal}$ in 
the goblet cells and epithelium of the duodenum, ileum, rectum, and lacteals (Figure $1 \mathrm{~A}-\mathrm{c}, \mathrm{A}-\mathrm{d}$, and A-e). In contrast, SA $2,3 \mathrm{Gal}$ receptors stained with MAAI lectin showed significant expression in the lacteals, but MAAI exhibited weak staining in both the goblet cells and ciliated epithelial cells (Figure 1A-c, A-d, and A-e). Strong MAAII lectin staining was mainly observed on the apical side of the ciliated epithelial cells (Figure 1B-c, B-d, and B-e).

The expression patterns of avian and human receptors were also defined in the kidney, as kidney cell lines are usually utilized for influenza viruses' isolation and propagation. The MAAI, MAAII, and SNA staining revealed that both avian and human receptors could be detected in the kidney of pika, but MAAII and SNA demonstrated much stronger staining in the epithelial cells from the glomeruli and proximal tubules. In contrast, SA $\alpha 2,3 \mathrm{Gal}$ rather than SA $\alpha 2,6 \mathrm{Gal}$ receptors were present in the epithelial cells from the distal tubules (Figure 1A-f and B-f).

In agreement with the findings in mouse (Mus muscu-

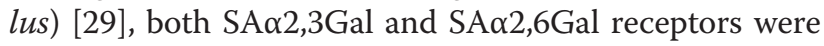
rare in the brain cells from pika (Figure 1A-g and B-g). In the liver, the SA $\alpha 2,6 \mathrm{Gal}$ and SA $\alpha 2,3 \mathrm{Gal}$ stained with MAAII lectin were predominant on the hepatocytes and sinusoid, while weak SA $2,3 \mathrm{Gal}$ signal stained with MAAI was found in the liver sinusoid, as well as on the membrane of Kupffer cells (Figure 1A-h and B-h).

In the heart, MAAI, MAAII and SNA displayed very strong staining in the cardiac myocytes (Figure $1 \mathrm{~A}-\mathrm{i}$ and $\mathrm{B}-\mathrm{i})$, suggesting that both $\alpha 2,6 \mathrm{Gal}$ and $\alpha 2,3 \mathrm{Gal}$ are widely expressed in the heart. The dominant expression of both avian and human receptors makes heart a very susceptible target for avian and human influenza viruses.

In the spleen, MAAI, MAAII, and SNA staining was detected in both the red and white pulps, but the SA $\alpha 2,6 \mathrm{Gal}$ signal was much more intense. A similar distribution pattern was also found in the lymphatic sheath around the artery in the white pulp regions (Figure $1 \mathrm{~A}-\mathrm{j}$ and $B-j)$.

\section{Pika could be infected with $\mathrm{H} 1, \mathrm{H} 3$, and $\mathrm{H} 5$ three subtypes of influenza $A$ viruses}

After ensuring that the animals were not $\mathrm{H} 1, \mathrm{H} 3$, or $\mathrm{H} 5$ sero-positive with hemagglutination inhibition (HI) assay, 118 pikas (100-170 g) were divided into four groups. Three groups were intranasally inoculated with $7 \times 10^{4}$ pfu of $\mathrm{H} 1, \mathrm{H} 3$, or $\mathrm{H} 5$ influenza virus, and the fourth group was inoculated with PBS as a control. At five days post infection, most influenza virus-infected pikas, except 10 animals infected with $\mathrm{H} 5 \mathrm{~N} 1$, did not display characteristic signs of illness, such as shivering, ruffled fur, and hunched posture. To investigate the infection of three different influenza viruses, we tested the viral M RNA in the lungs at 5 days post infection (d.p.i.) using RT-PCR. The three different influenza viruseschallenged groups $(\mathrm{H} 1 \mathrm{~N} 1, \mathrm{H} 3 \mathrm{~N} 2$, and $\mathrm{H} 5 \mathrm{~N} 1)$ presented different infectivities $(30 \%, 21.4 \%$, and $43.3 \%$, respectively) (Table 1).

To confirm the virus infection and viral antigen distribution in the tissues of the infected animals, immunohistochemical analyses with monoclonal antibody against M1 were performed. The expression of viral antigen M1 could be detected in the lung tissues of H1N1-, H5N1-, and H3N2-infected pikas, which were confirmed by the RTPCR assay. The M1 viral antigen could be found in both ciliated and alveolar epithelial cells all over the lung in both the H1N1- (Figure 2B) and H5N1-infected pikas (Figure 2C), while M1 antigen could only be detected focally in the epithelial cells of a few bronchi and alveolar cells in the H3N2-infected animals (Figure 2D). Additionally, influenza virus antigen staining revealed that M1 antigens were mainly observed in the Purkinje cells, some of the largest neurons that coordinate voluntary movements in the brain of H5N1-infected pikas (Figure 2F). Taken together, pika could be infected by different subtypes of influenza A viruses at high doses, though infectivity and infection tropism in the lungs were various.

\section{Pathological changes in the organs and tissues of influenza viruses-infected pika}

To elucidate any pathological damage that may occur in influenza A viruses-infected pikas, various tissues, including the lung, spleen, kidney, brain, liver, intestine, and trachea, were collected from pikas infected with avian $\mathrm{H} 5 \mathrm{~N} 1$, human $\mathrm{H} 1 \mathrm{~N} 1$, and $\mathrm{H} 3 \mathrm{~N} 2$ influenza viruses and stained with $\mathrm{H} \& \mathrm{E}$. We observed various levels of severity in the virus-infected lungs (Figure 3). Compared to the PBS control group (Figure 3A), the alveolar structure appeared normal, and a few small bronchi and terminal bronchioles were infiltrated with lymphocytic cells in the H3N2-infected lung tissue (Figure 3D). Slight pathological changes, such as alveolar capillary congestion, edema, and thickening of the alveolar interstitium with local lymphocytic infiltrations, were observed in the H1N1-infected lungs. Even though the alveolar cavity became smaller, no exudation within the alveolar space was found (Figure 3B). In contrast, serious damage, including a large area of alveolar capillary hyperemia, edema,

$\begin{aligned} & \text { Table } 1 \text { Detection of viruses among the infected pikas } \\
& \text { with RT-PCR analysis }\end{aligned}$
\begin{tabular}{lllll}
\hline Virus strains & No. of pika & PCR positive & Infectivity (\%) \\
\hline A/WSN/1933 & 30 & 9 & 30.0 \\
A/Jiangxi/262/2005 & 28 & 6 & 21.4 \\
A/great black-headed gull// & 30 & 13 & 43.3 \\
Qinghai/1/2009 & & & \\
PBS & 30 & 0 & 0 \\
\hline
\end{tabular}



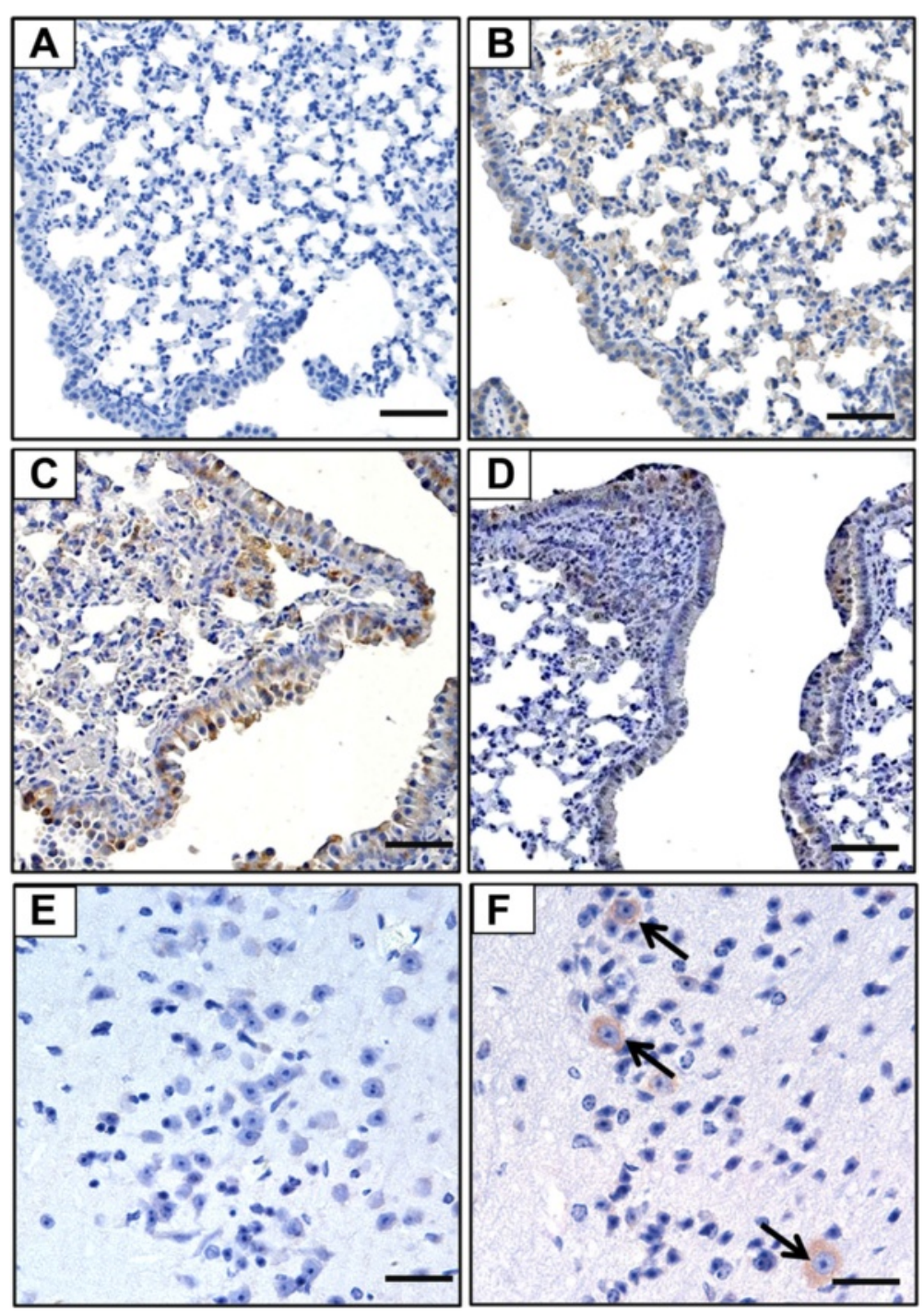

Figure 2 Detection of viral antigen M1 protein in the lung of H1N1- and H3N2-infected pikas, as well as in the lung and brain from H5N1-infected pikas. Immunohistochemical analyses of viral antigen distribution in the tissues of pika using a monoclonal antibody against $\mathrm{M} 1$ protein in (A) Uninfected lung, (B) H1N1-infected lung, (C) H5N1-infected lung, (D) H3N2-infected lung, (E) Uninfected brain, and (F) H5N1-infected brain. Arrows denote the M1-positive Purkinje cells. M1 is expressed on the membrane and in the cytoplasm of epithelial cells of the small bronchi, terminal bronchioles, and alveolar cells. Bar, $100 \mu \mathrm{m}$.

alveolar collapse, and lymphocytic infiltrations, was present in the lungs of H5N1-infected pika (Figure 3C).

Unlike the severity observed in the lung tissue, ileum and livers from H5N1-infected pikas showed no significant alterations (Figure 4D, F). Similarly, obvious pathogenic changes were not found in other organs, including the trachea, kidney, liver, ileum, brain, and spleen in the H1N1- and H3N2-infected pikas (data not shown). Notably, increases in the desquamation of apoptotic ciliated epithelial cells in tracheas (Figure 4B), lymphocytic infiltrations around glomerular afferent arterioles in the kidney (Figure 4H), apoptotic Purkinje cells in the cerebellum (Figure 4J), and expansion of the white pulp in the spleen (Figure 4L) were obvious in the
H5N1-infected pikas. Apoptotic progress observed in Purkinje neurons is the result of H5N1 infection and replication, which was demonstrated by immunostaining for the M1 antigen. The pathological changes were also the cause of the signs of illness in pika. These results demonstrate that a high-dose infection of influenza A viruses is able to induce variant viral pneumonia and multi-organ abnormalities in wild pika.

\section{Discussion}

Following the outbreak of severe H5N1 HPAI in wild birds $[21,22]$, plateau pika infections with two distinct lineages, a mixed Vietnam $\mathrm{H} 5 \mathrm{~N} 1$ virus lineage and a wild bird Qinghai-like H5N1 virus lineage, were reported 

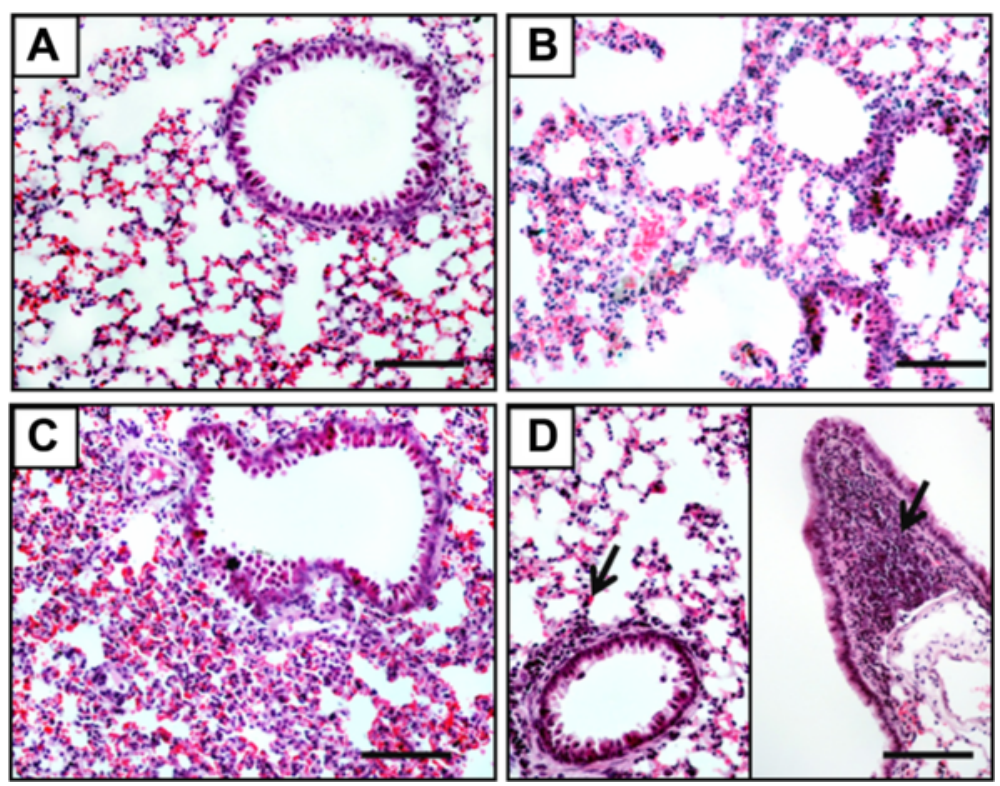

Figure 3 Histopathological analyses of lungs from different influenza virus-infected or uninfected pikas by H \& E staining. (A) The uninfected lung serves as the normal control ( $n=5$, control group; $n=5$, infected group). (B) Slight alveolar capillary congestion, edema, and thickening of the interstitium of alveolar cells with local lymphocyte infiltration were observed in H1N1-infected lung; no exudation within the alveolar lumen was found in the H1N1-infected lung. (C) Alveolar capillary hyperemia, edema, and alveolar collapse with lymphocyte infiltration were found throughout of the H5N1-infected lung. (D) The alveolar structure was normal, with only a few small bronchi and terminal bronchioles with lymphocytic infiltration in the H3N2-infected lung (arrows). Bar, $100 \mu \mathrm{m}$.

in 2007 around Qinghai Lake [24]. Considering that wild birds share the same habitats with pika and that some small birds often enter into pika caves for feeding, critical questions were raised concerning whether influenza virus is highly pathogenic to pika and whether pika could act as an influenza virus-carrier. To answer these questions, we first investigated the influenza virus receptor distribution in plateau pika. One interesting finding was that SA $2,6 \mathrm{Gal}$ linked saccharide receptors are present in high amounts in all of the tested organs and tissues, especially in the lungs. The wide presence of SA $22,6 \mathrm{Gal}$ linked receptors may facilitate the replication of the human origin influenza A viruses in pika. It also suggests that pika could be more important as an intermediate host for the generation of influenza viruses with increased binding affinity to $\mathrm{SA} \alpha 2,6 \mathrm{Gal}$ and $\mathrm{SA} \alpha 2,3 \mathrm{Gal}$ receptors.

We also demonstrated that pika could be experimentally infected with various subtypes of influenza A viruses, e.g., human viruses $\mathrm{H} 1 \mathrm{~N} 1, \mathrm{H} 3 \mathrm{~N} 2$, and the avian virus H5N1. However, virus replication in the lung tissue and viral infectivity differed among these three subtypes, which is consistent with the predominant receptor expression in the trachea and lung and the receptor preferences of human $\mathrm{H} 1 \mathrm{~N} 1$ and $\mathrm{H} 3 \mathrm{~N} 2$ and avian $\mathrm{H} 5 \mathrm{~N} 1$. Dominant expression of SA $\alpha 2,3 \mathrm{Gal}$ in both the trachea and lung facilitates the infection and replication of H5N1 avian influenza virus. In contrast, SA $\alpha 2,6 \mathrm{Gal}$ was distributed mainly in the lungs rather than in the trachea. This explains why much more viral antigen M1positive cells were stained in the lung of avian receptorpreferring H5N1-infected pikas, and why a higher infection rate occurred in the avian $\mathrm{H} 5 \mathrm{~N} 1$-challenged group than those in the human receptor-preferring H1N1 and H3N2 infected ones.

Furthermore, H1N1, H3N2, and H5N1 caused different types of damage in the lungs of infected pikas. Similar to the H5N1-infected rabbits (Oryctolagus cuniculus) [24], H1N1-and H3N2-infected pikas displayed mild pathological changes with lymphocytic infiltrations in the lung. In contrast, serious damage, such as alveolar capillary hyperemia, edema, alveolar collapse, and lymphocytic infiltrations, were observed in the H5N1-infected group. Different degrees of lung damage were correlated with the number of viral antigen M1-positive cells, as well as viral infectivity.

In contrast to the lung tissue, the presence of avian receptor SA 2,3Gal in the trachea, lung, kidney, and spleen may facilitate $\mathrm{H} 5 \mathrm{~N} 1$ attachment, which in turn caused the lesions and lymphocytic infiltrations in these tissues. We also demonstrated that avian $\mathrm{H} 5 \mathrm{~N} 1$ infected the pika central neuron system (CNS) by attacking Purkinje neurons in the cerebellum, which is in consistent with H5N1 infection in ferret (Mustela putorius furo) and crow (Corvus) [36,37]. These results demonstrate that pika is more susceptible to H5N1 avian influenza virus, and the pathogenic response of pika to influenza viruses 

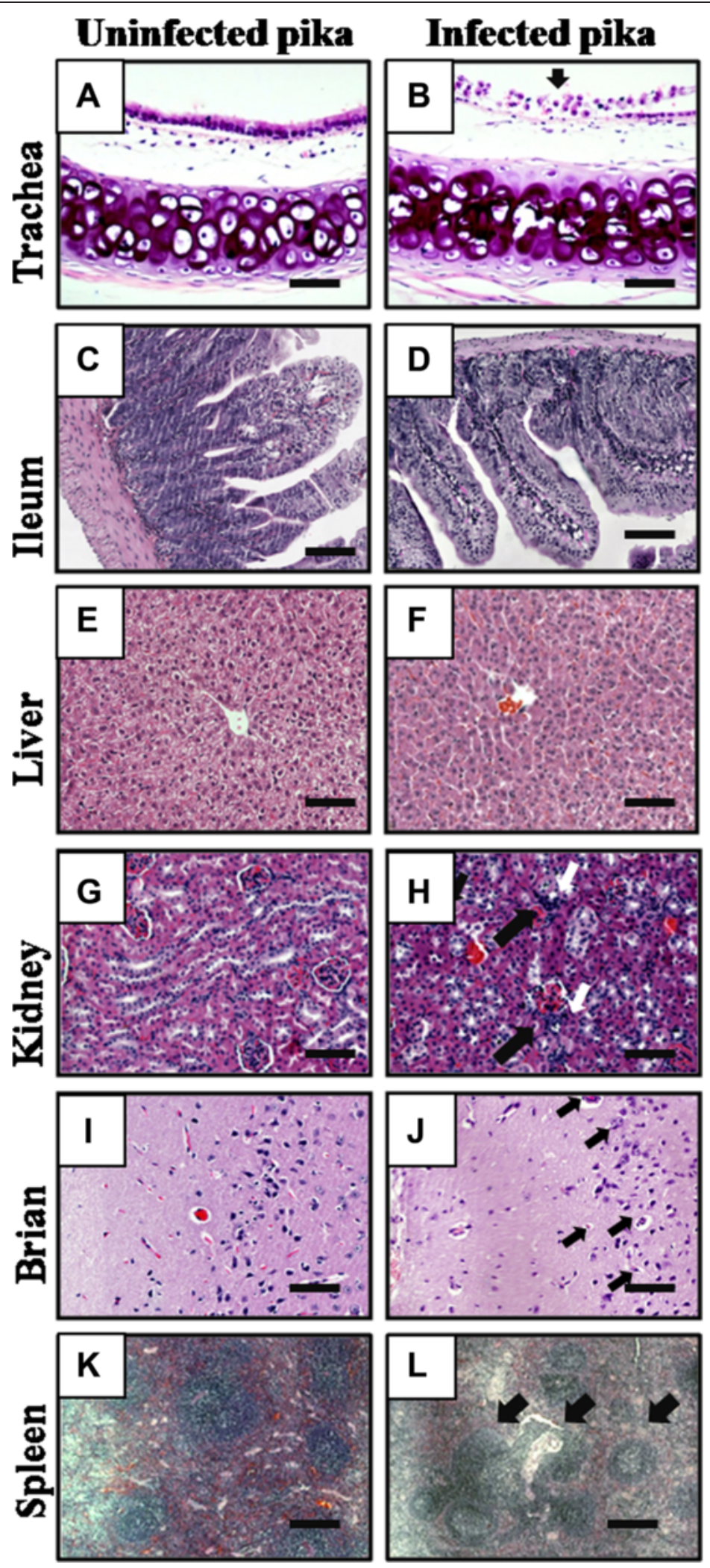

Figure 4 (See legend on next page.) 
(See figure on previous page.)

Figure 4 Histopathology in different tissues of H5N1-infected pika. (A, C, E, G, I, K): Representative specimen from the uninfected control group ( $n=5$, control group; $n=5$ per infected group). (B, D, F, H, J, L): Representative specimen from the H5N1-infected group. (A) Uninfected Trachea. (B) Desquamation of the apoptotic tracheal epithelial cells was observed in the virus-infected trachea (arrow). (C) Uninfected ileum. (D) No notable abnormalities were observed in the ileum from the infected pika. (E) Uninfected liver. (F) No significant abnormalities were observed in the liver from the infected pika. (G) Uninfected kidney. (H) Lymphocytic infiltrations around glomerular afferent arterioles in the kidney (arrows). (I) Uninfected cerebellum. (J) Apoptotic Purkinje cells were observed in the infected cerebellum (arrows). (K) Uninfected spleen. (L) Enlarged white pulps were observed in the virus-infected spleen (arrows). Bar, $50 \mu \mathrm{m}$.

is subtype- or strain-dependent, though the mechanism involved needs to be further explored.

Taken together, we demonstrated that pika is capable of being an influenza host, though human origin H1N1 and H3N2 viruses had relative low replication efficiency in the lung than avian H5N1. As the influenza virus continues to circulate within the pika population, the influenza viruses that have been found in wild plateau pika are most likely transmitted from wild birds. The presence of SA $\alpha 2,6 \mathrm{Gal}$ and $\mathrm{SA} \alpha 2,3 \mathrm{Gal}$ receptors in many organs suggest that pika could act as an efficient virus reservoir and possible mixing vessels. Consequently, we would advise that monitoring of viruses among the wild bird populations in the Qinghai-Tibet plateau should also be extended to monitor the wild pika.

\section{Methods}

\section{Virus strains and virus amplification}

Influenza viruses A/WSN/1933 (H1N1) and A/Jiangxi/ 262/2005 (H3N2) were amplified in MDCK cells; A/ great black-headed gull/Qinghai/1/2009 (H5N1) was propagated in 10 day-old SPF embryonated eggs. Viral titers were determined by plaque assays. Briefly, confluent MDCK cells in 12-well plates were washed twice with PBS and then infected with $500 \mu \mathrm{l}$ virus in DMEM (GIBCO, Grand Island, NY) at a concentration range from $10^{-1}$ to $10^{-6}$ for 1 hour (h) at $37^{\circ} \mathrm{C}$ with gentle shaking. The inocula were then removed, and the cells were washed twice and overlaid with DMEM containing $1 \%$ agarose (Promega, Madison, WI) and $5 \mu \mathrm{g} / \mathrm{ml}$ TPCK treated trypsin. After overlays are solidified, the cells were incubated at $37^{\circ} \mathrm{C}$ with $5 \% \mathrm{CO}_{2}$ for 72 hours (hrs). Cells were then fixed with $4 \%$ formaldehyde in PBS for $1 \mathrm{~h}$ and stained with neutral red at room temperature (RT). All experiments with the H5N1 virus were performed in a biosafety level 3 laboratory.

\section{Lectin detection of SAa2,3Gal and SAa2,6Gal}

Sections of various tissues were deparaffinized and rehydrated as described below for $\mathrm{H} \& \mathrm{E}$ staining. After being washed in distilled water for 5 minutes at RT, sections were heat-treated with the antigen-retrieval solution (citrate buffer, $\mathrm{pH}$ 6.0) four times, each time for 4 minutes. Before being stained with lectins, sections were blocked with $10 \%$ horse serum (GIBCO, Grand Island, NY) for 10 minutes at RT. Tissue sections were incubated with specific FITC-labeled SNA lectin to detect SA $\alpha 2,6$ Gal receptor (Vector Laboratories, Burlingame, CA) and biotinylated MAAI and MAAII lectins for SA $\alpha 2,3 \mathrm{Gal}$ (Vector Laboratories, USA ) for $2 \mathrm{hrs}$ at $37^{\circ}$ C. After being washed with PBS three times, the sections were incubated with PE-conjugated streptavidin (Sigma, Santa Clara, CA) for 2 hrs at RT. The sections were washed in PBS three times to remove unbound streptavidin and then counterstained with 4,6-diamidino-2-phenylindole (DAPI) (Roche, Switzerland).

\section{Animal infection with influenza A viruses}

The pika animals in this experiment were caught in regions around Haibei Alpine Grassland Ecosystem Research Station in Qinghai Province, China. Sera collected from pikas via the lateral tail veins were tested by $\mathrm{HI}$ and neutralization assays with $\mathrm{H} 1, \mathrm{H} 3$, and $\mathrm{H} 5$ influenza viruses to rule out influenza virus sero-positive animals. Female adult pikas with body masses of approximately 100-170 g were selected and kept in captivity for 7 days to adapt to the environment before being infected with influenza viruses. Food and water were provided every day. The animals were anesthetized with pentobarbital sodium salt (Sigma, $40 \mathrm{mg} / \mathrm{kg}$ ) via intraperitoneal injection and intranasally infected with $7.0 \times 10^{4}$ pfu of $\mathrm{H} 5 \mathrm{~N} 1, \mathrm{H} 1 \mathrm{~N} 1$, or H3N2 virus. Five days post virus infection, pikas were sacrificed, and tissues, including the heart, liver, spleen, half of the lung, trachea, kidney, brain, and intestine, were collected and stored in neutral formalin; the other half of the lungs were frozen in liquid nitrogen for RNA extraction.

The Ethics Committee for animal experimentation of the Institute of Microbiology, Chinese Academy of Sciences, approved all of the animal experiments (Registry No. CASPMI2008001).

\section{Immunohistochemistry (IHC) detection of influenza A virus $\mathrm{M} 1$ protein}

Immunohistochemical staining was performed as described below. Reagents used in this study were described as follows: mouse anti-M1 monoclonal antibody (1:200 dilution, a gift from Professor Wenjun Liu, Institute of Microbiology, 
CAS.), biotinylated goat-anti-mouse secondary antibody (1:200 dilution, Vector Laboratories, USA), and strepavidinconjugated HRP (1:200 dilution, Jackson Immuno Research, West Grove, PA).

\section{Viral RNA extraction and RT-PCR}

To identify infection in experimental pikas, viral RNA from the lung of each pika was extracted as described in the RNeasy Mini kit (Qiagen, Hilden, Germany). Briefly, $30 \mathrm{mg}$ lung tissue was disrupted and homogenized in $600 \mu \mathrm{l}$ RLT buffer supplied in the kit, and the nucleic acids were eluted with $30 \mu \mathrm{l}$ nuclease-free water. Lungs from uninfected pikas were used as the negative control. Viral cDNA was immediately obtained using a Reverse Transcription System kit (Promega, Madison, WI) with primer Uni12 5' -AGCAAAAGCAGG-3'. Amplification of the M segment was performed with primers UMF 5 '-TATTCGT CTC AGGGAGCAAAAGCAGGTAG-3', and UMR5' ATATCGTCTCGTATTAGTAGAAACAAGGTAGTTT TT-3'.

\section{Morphological and histological analyses}

Tissues collected from pikas were dehydrated with increasing concentrations of ethanol, vitrified by dimethylbenzene, and then embedded in paraffin. After being embedded, the tissues were sectioned with a rotary microtome (Hestion ERM3000, China). Sections (5 $\mu \mathrm{m}$-thicknesses) were deparaffinized in dimethylbenzene, rehydrated using graded ethanol, and stained with $\mathrm{H}$ \& $\mathrm{E}$ for histopathological examination. Before being mounted, sections were dehydrated and vitrified. Mounted slides were observed under a fluorescence microscope (IX71 Olympus Co., Ltd., Tokyo) and photographed with a Nikon DXM1200F (Nikon Co., Tokyo).

\section{Additional files}

Additional file 1: Figure S1. Expression of SAa2,3Gal (MAAl, MAAll lectin) and SAa2,6Gal (SNA lectin) receptors in duck duodenum and human trachea. (A) Expression of SAa2,3Gal (red) and SAa2,6Gal (green) receptors were detected by MAAI and SNA lectins staining in the contrast to nuclear staining (blue) with DAPI, and presented with composite confocal images in human trachea and duck duodenum. Bar, 200 um. (B) Expression of SAa2,3Gal (red) receptors were revealed by MAAll staining in the distinguish with nuclear staining (blue) with DAPI. Different organs labeling is the same order with those in supplementary Figure $1 \mathrm{~A}$. Bar, $200 \mu \mathrm{m}$. SAa2,3Gal receptors stained with MAAl and MAAll lectins are exclusively present in the duck duodenum, whereas, human trachea only shows the expression of SAa2,6Gal receptors.

Additional file 2: Figure S2. Selective presence of SAa2,3Gal (MAAI, MAAll lectin) and SAa2,6Gal (SNA lectin) receptors in different organs of pika. Expression of SAa2,3Gal (MAAl, MAAll, red) and SAa2,6Gal (SNA, green) receptors were detected by MAAl, MAAll and SNA lectins staining in the contrast to nuclear staining (blue) with DAPI, and presented with composite confocal images in many pika organs including (A) trachea, lung, duodenum, ileum, rectum, and others (B) kidney, brain, liver, heart and spleen indicated above. Bar, 100 m. SAa2,3Gal (MAAl, MAAll lectin) and SAa2,6Gal (SNA lectin) receptors are selectively present in these organs. SAa2,6Gal receptors are widely expressed in the lung, kidney, liver, spleen, duodenum, ileum, rectum and heart, whereas SAa2,3Gal receptors are dominant in the trachea, lung, ileum, kidney, liver, heart and spleen.

\section{Competing interests}

The authors declare that they have no competing interests.

\section{Authors' contributions}

YL participated in the experiment design, carried out all experiments and subsequent data analysis. HX participated in the conception and design of the study, carried out data analysis and interpretation, and drafted the manuscript. $\mathrm{CH}$ took part in the immunoassay and histological analysis. HS and HW participated in animal experiments. LL participated in the collection of animals. JS contributed to the study design and data interpretation. WL, JM and DL participated in study design and manuscript revision. GFG, XL and JY participated in study design and coordination, data analysis, as well as revision of manuscript. All authors read and approved the final manuscript.

\section{Acknowledgements}

This work was supported by the Ministry of Science and Technology of China (MOST) 973 Project (Grant No. 2010CB530303), Youth Innovation Promotion Association, CAS, and US Civilian Research \& Development Foundation from the National Institute of Allergy and Infectious Diseases (No. CHB1-31057-BE-11). George F. Gao is a leading principal investigator of the National Natural Science Foundation of China (NSFC) Innovative Research Group (Grant No. 81321063). We thank Joel Heywood and Guangwen Lu for critical reading and English editing.

\section{Author details}

${ }^{1}$ CAS Key Laboratory of Pathogenic Microbiology and Immunology, Institute of Microbiology, Chinese Academy of Sciences, Beijing 100101, China.

${ }^{2}$ Laboratory of Protein Engineering and Vaccines, Tianjin Institute of Industrial Biotechnology, Chinese Academy of Sciences, Tianjin 300308, China. ${ }^{3}$ State Key Laboratory of the Agro-Biotechnology, College of Biological Sciences, China Agricultural University, Beijing 100193, China. ${ }^{4}$ College of Veterinary Medicine, China Agricultural University, Beijing 100193, China. ${ }^{5}$ Northwest Institute of Plateau Biology, Chinese Academy of Sciences, Xining 810008, China. ${ }^{6}$ Network Information Center, Institute of Microbiology, Chinese Academy of Sciences, Beijing 100101, China. ${ }^{7}$ Research Network of Immunity and Health, Beijing Institutes of Life Science, Chinese Academy of Sciences, Beijing 100101, China. ${ }^{8}$ Office of Director-General, Chinese Center for Disease Control and Prevention, Beijing 102206, China.

Received: 23 September 2014 Accepted: 27 March 2015 Published online: 14 April 2015

\section{References}

1. Kim JK, Negovetich NJ, Forrest HL, Webster RG. Ducks: the "Trojan horses" of H5N1 influenza. Influenza Other Respir Viruses. 2009;3:121-8.

2. Tong S, Li Y, Rivailler P, Conrardy C, Castillo DA, Chen LM, et al. A distinct lineage of influenza A virus from bats. Proc Natl Acad Sci U S A. 2012;109:4269-74

3. Tong S, Zhu X, Li Y, Shi M, Zhang J, Bourgeois M, et al. New world bats harbor diverse influenza A viruses. PLoS Pathog. 2013;9:e1003657.

4. Wu Y, Wu Y, Tefsen B, Shi Y, Gao GF. Bat-derived influenza-like viruses H17N10 and H18N11. Trends Microbiol. 2014;22:183-91.

5. Sun X, Shi Y, Lu X, He J, Gao F, Yan J, et al. Bat-derived influenza hemagglutinin $\mathrm{H} 17$ does not bind canonical avian or human receptors and most likely uses a unique entry mechanism. Cell Rep. 2013;3:769-78.

6. Zhu X, Yu W, McBride R, Li Y, Chen LM, Donis RO, et al. Hemagglutinin homologue from H17N10 bat influenza virus exhibits divergent receptorbinding and pH-dependent fusion activities. Proc Natl Acad Sci U S A. 2013;110:1458-63.

7. Li Q, Sun X, Li Z, Liu Y, Vavricka CJ, Qi J, et al. Structural and functional characterization of neuraminidase-like molecule N10 derived from bat influenza A virus. Proc Natl Acad Sci U S A. 2012;109:18897-902.

8. Zhu XY, Yang H, Guo Z, Yu WL, Carney PJ, Li Y, et al. Crystal structures of two subtype N10 neuraminidase-like proteins from bat influenza A viruses reveal a diverged putative active site. Proc Natl Acad Sci U S A. 2012;109:18903-8. 
9. Subbarao K, Klimov A, Katz J, Regnery H, Lim W, Hall H, et al. Characterization of an avian influenza A (H5N1) virus isolated from a child with a fatal respiratory illness. Science. 1998;279:393-6.

10. Beigel JH, Farrar J, Han AM, Hayden FG, Hyer R, de Jong MD, et al. Avian influenza A (H5N1) infection in humans. N Engl J Med. 2005;353:1374-85.

11. Yuan J, Zhang L, Kan X, Jiang L, Yang J, Guo Z, et al. Origin and molecular characteristics of a novel 2013 avian influenza A(H6N1) virus causing human infection in Taiwan. Clin Infect Dis. 2013;57:1367-8.

12. Skowronski DM, Tweed SA, Petric M, Booth T, Li Y, Tam T. Human illness and isolation of low-pathogenicity avian influenza virus of the H7N3 subtype in British Columbia, Canada. J Infect Dis. 2006;193:899-900. author reply 900-891.

13. Eames KT, Webb C, Thomas K, Smith J, Salmon R, Temple JM. Assessing the role of contact tracing in a suspected H7N2 influenza A outbreak in humans in Wales. BMC Infect Dis. 2010;10:141.

14. Fouchier RA, Schneeberger PM, Rozendaal FW, Broekman JM, Kemink SA, Munster $\mathrm{V}$, et al. Avian influenza A virus (H7N7) associated with human conjunctivitis and a fatal case of acute respiratory distress syndrome. Proc Natl Acad Sci U S A. 2004;101:1356-61.

15. Peiris M, Yuen KY, Leung CW, Chan KH, Ip PL, Lai RW, et al. Human infection with influenza H9N2. Lancet. 1999;354:916-7.

16. Arzey GG, Kirkland PD, Arzey KE, Frost M, Maywood P, Conaty S, et al. Influenza virus A (H10N7) in chickens and poultry abattoir workers, Australia. Emerg Infect Dis. 2012;18:814-6.

17. Chen $\mathrm{H}$, Yuan $\mathrm{H}$, Gao R, Zhang J, Wang D, Xiong Y, et al. Clinical and epidemiological characteristics of a fatal case of avian influenza A H10N8 virus infection: a descriptive study. Lancet. 2014;383:714-21.

18. Zhang T, Bi Y, Tian H, Li X, Liu D, Wu Y, et al. Human infection with influenza virus $A(\mathrm{H} 10 \mathrm{~N} 8)$ from live poultry markets, China, 2014. Emerg Infect Dis. 2014;20:2076-9.

19. World Health Organization. Cumulative number of confirmed human cases for avian influenza A (H5N1) reported to WHO, 2003-2014. http://www.who. int/influenza/human_animal_interface/EN_GIP_20140124CumulativeNumber H5N1cases.pdf; 24 January 2014.

20. World Health Organization. Confirmed human cases of avian influenza A (H7N9) reported to WHO. http://www.who.int/influenza/human_animal_ interface/influenza_h7n9/18_reportwebh7n9number_20140714.pdf?ua=1; 14 July 2014.

21. Liu J, Xiao H, Lei F, Zhu Q, Qin K, Zhang XW, et al. Highly pathogenic H5N1 influenza virus infection in migratory birds. Science. 2005;309:1206

22. Chen H, Smith GJ, Zhang SY, Qin K, Wang J, Li KS, et al. Avian flu: H5N1 virus outbreak in migratory waterfowl. Nature. 2005;436:191-2.

23. World Health Organization. H5N1 highly pathogenic avian influenza: Timeline of major events. http://www.who.int/influenza/ human_animal_interface/H5N1_avian_influenza_update20121210.pdf; 10 December 2013

24. Zhou JY, Sun WB, Wang JH, Guo JQ, Yin W, Wu NP, et al. Characterization of the $\mathrm{H} 5 \mathrm{~N} 1$ highly pathogenic avian influenza virus derived from wild pikas in China. J Virol. 2009:83:8957-64.

25. Gagneux P, Cheriyan M, Hurtado-Ziola N, van der Linden EC, Anderson D, McClure $\mathrm{H}$, et al. Human-specific regulation of alpha 2-6-linked sialic acids. J Biol Chem. 2003;278:48245-50.

26. Matrosovich MN, Matrosovich TY, Gray T, Roberts NA, Klenk HD. Human and avian influenza viruses target different cell types in cultures of human airway epithelium. Proc Natl Acad Sci U S A. 2004;101:4620-4.

27. Ibricevic A, Pekosz A, Walter MJ, Newby C, Battaile JT, Brown EG, et al. Influenza virus receptor specificity and cell tropism in mouse and human airway epithelial cells. J Virol. 2006;80:7469-80.

28. Kuchipudi SV, Nelli R, White GA, Bain M, Chang KC, Dunham S. Differences in influenza virus receptors in chickens and ducks: Implications for interspecies transmission. J Mol Genet Med. 2009;3:143-51.

29. Ning ZY, Luo MY, Qi WB, Yu B, Jiao PR, Liao M. Detection of expression of influenza virus receptors in tissues of BALB/C mice by histochemistry. Vet Res Commun. 2009;33:895-903

30. Jayaraman A, Chandrasekaran A, Viswanathan K, Raman R, Fox JG, Sasisekharan R. Decoding the distribution of glycan receptors for human-adapted influenza A viruses in ferret respiratory tract. PLoS One. 2012;7:e27517.

31. Kim M, Yu JE, Lee JH, Chang BJ, Song CS, Lee B, et al. Comparative analyses of influenza virus receptor distribution in the human and mouse brains. J Chem Neuroanat. 2013:52:49-57.
32. Zeng H, Goldsmith CS, Maines TR, Belser JA, Gustin KM, Pekosz A, et al. Tropism and infectivity of influenza virus, including highly pathogenic avian $\mathrm{H} 5 \mathrm{~N} 1$ virus, in ferret tracheal differentiated primary epithelial cell cultures. J Virol. 2013;87:2597-607.

33. Trebbien R, Larsen LE, Viuff BM. Distribution of sialic acid receptors and influenza A virus of avian and swine origin in experimentally infected pigs. Virol J. 2011:8:434

34. Shinya K, Ebina M, Yamada S, Ono M, Kasai N, Kawaoka Y. Avian flu: influenza virus receptors in the human airway. Nature. 2006;440:435-6.

35. Nelli RK, Kuchipudi SV, White GA, Perez BB, Dunham SP, Chang KC. Comparative distribution of human and avian type sialic acid influenza receptors in the pig. BMC Vet Res. 2010;6:4.

36. Plourde JR, Pyles JA, Layton RC, Vaughan SE, Tipper JL, Harrod KS. Neurovirulence of H5N1 infection in ferrets is mediated by multifocal replication in distinct permissive neuronal cell regions. PLoS One. 2012;7:e46605.

37. Tanimura N, Tsukamoto K, Okamatsu M, Mase M, Imada T, Nakamura K, et al. Pathology of fatal highly pathogenic $\mathrm{H} 5 \mathrm{~N} 1$ avian influenza virus infection in large-billed crows (Corvus macrorhynchos) during the 2004 outbreak in Japan. Vet Pathol. 2006:43:500-9.

\section{Submit your next manuscript to BioMed Central and take full advantage of:}

- Convenient online submission

- Thorough peer review

- No space constraints or color figure charges

- Immediate publication on acceptance

- Inclusion in PubMed, CAS, Scopus and Google Scholar

- Research which is freely available for redistribution 